\title{
Self-consistent Keldysh approach to quenches in weakly interacting Bose-Hubbard model
}

\author{
N. Lo Gullo ${ }^{1,2}$ and L. Dell'Anna ${ }^{1}$ \\ ${ }^{I}$ Dipartimento di Fisica e Astronomia "G. Galilei”, Università degli Studi di Padova, and CNISM, Padova, Italy \\ ${ }^{2}$ Dipartimento di Fisica, Università degli Studi di Milano, Milano, Italy
}

\begin{abstract}
We present a non-equilibrium Green's functional approach to study the dynamics following a quench in weakly interacting Bose-Hubbard model (BHM). The technique is based on the self-consistent solution of a set of equations which represents a particular case of the most general set of Hedin's equations for the interacting single-particle Green's function. We use the ladder approximation as a skeleton diagram for the two-particle scattering amplitude useful, through the self-energy in the Dyson equation, for finding the interacting singleparticle Green's function. This scheme is then implemented numerically by a parallelized code. We exploit this approach to study the correlation propagation after a quench in the interaction parameter, for one (1D) and two (2D) dimensions. In particular, we show how our approach is able to recover the crossover from ballistic to diffusive regime by increasing the boson-boson interaction. Finally we also discuss the role of a thermal initial state on the dynamics both for 1D and 2D BHMs, finding that surprisingly at high temperature a ballistic evolution is restored.
\end{abstract}

PACS numbers:

\section{INTRODUCTION}

The study of out-of-equilibrium quantum systems has received quite some attention in recent years and many efforts have been devoted to understand properties of thermalization or relaxation towards equilibrium in the quantum regime ${ }^{1 / 3}$, transpor ${ }^{4}$, dynamical phase transition $s^{2518}$, ergodic and nonergodic quantum systems ${ }^{3}$. The quickly developing field of ultracold atomic gases offers the possibility of manipulating and controlling complex quantum systems with very high accuracy. This tunability has undoubtedly increased the interest in studying the dynamics in such systems due to the possibility of experimentally testing theoretical predicitons and offering new research perspectives 9 -11. The Bose-Hubbard model (BHM) is one of the most studied system in this context, because of its rich phase diagram $\frac{1213}{213}$ and because it describes faithfully the dynamics of ultracold atomic bosons in optical lattices $\frac{813}{15}$, and therefore it is relevant for experimental investigations. In order to theoretically study the BHM generally one can resort to the following numerical tools: exact numerical diagonalization ${ }^{816}$ and time dependent density matrix renormalization group (t-DMRG) approach 17 .

A very powerful method for non-equilibrium systems is the Keldysh-Green's functional approach developed to describe the dynamics of a quantum system without the assumption that the system relaxes towards a steady state ${ }^{18119}$. This technique can be formulated either in the form of equations of motion for the single-particle Green's function or as a manybody perturbation approach, eventually resumming some of the diagrams appearing in the series. For the single particle Green's function, and for a two-body interaction Hamiltonian, it is possible to define a closed set of equations, whose iterative solution is the solution to the initial problem.20. This set of equations has been named after Hedin the Hedin's equations. The iterative solution of such equations is obviously found by means a proper (depending upon the problem at hand) numerical approach. Hedin's equations are nevertheless difficult to be implemented because of the appearance of a functional derivative in the equation for the vertex function. One would thus need to find a way to calculate it analytically at different orders and then insert it into the numerical iteration. This process is nevertheless rather complicated and often the way out is to drop this equation thus effectively keeping the bare vertex at all iteration steps $\frac{20}{20}$.

However it is possible to recast Hedin's equations in a different, but fully equivalent, set of equations as shown by Starke and Kresse in their work ${ }^{21}$. The idea behind this is to exploit the fact that, for a two-body interaction Hamiltonian, the time evolution of the interacting single-particle Green's function is related only to the two-body Green's function. It is then possible to write a set of equations for both single- and two- particle Green's functions and then solve it iteratively. In our work we apply a slightly different version of this idea to study the dynamics of a BHM following a sudden quench in the boson-boson interaction strenght. Our approach is fully consistent with the work of Starke and Kresse ${ }^{21}$ and the only difference is that we will use the two-particle scattering amplitude instead of the two-particle Green's function.

The remainder of the paper is organized as follows: in Sec. II] we describe the self-consistent methods and discuss their properties, limitation and potentiality, in Sec. [III we introduce the perturbative theory used to study the dynamics of the weakly interacting Bose Hubbard model (BHM), whereas in Sec. IV we describe the corresponding iterative scheme used to find the time-dependent single particle Green's functions and its relation to the previously introduced schemes. Finally, in Secs. VI and VII we present a study of quenches in one and two dimensional BHM for different paramenters of the system such as initial chemical potential, final interaction, temperature, and dimensionality.

\section{SELF-CONSISTENT METHODS}

In this section we briefly review self-consistent methods which allow one to calculate the single-particle Green's function for an interacting many-body system with a two-body interaction. To fix the ideas we will consider from now a 
bosonic system described by the Hamiltonian:

$$
\begin{aligned}
\hat{\mathcal{H}} & =\hat{\mathcal{H}}_{0}+\hat{\mathcal{V}}(t) \\
\hat{\mathcal{H}}_{0} & =-\frac{\hbar^{2}}{2 m} \int d \mathbf{x} \hat{\phi}^{\dagger}(\mathbf{x}, t) \Delta \hat{\phi}(\mathbf{x}, t) \\
& +\int d \mathbf{x} v(\mathbf{x}) \hat{\rho}(\mathbf{x}, \mathbf{t}) \\
\hat{\mathcal{V}}(t) & =\frac{1}{2} \int d \mathbf{x} d \mathbf{x}^{\prime} u\left(\left|\mathbf{x}-\mathbf{x}^{\prime}\right|, t\right) \\
& \times \hat{\phi}^{\dagger}(\mathbf{x}, \mathbf{t}) \hat{\phi}^{\dagger}\left(\mathbf{x}^{\prime}, \mathbf{t}\right) \hat{\phi}\left(\mathbf{x}^{\prime}, \mathbf{t}\right) \hat{\phi}(\mathbf{x}, \mathbf{t}) .
\end{aligned}
$$

where $\hat{\phi}(\mathbf{x}, t)$ and $\hat{\phi}^{\dagger}(\mathbf{x}, t)$ are bosonic field operators satisfying commutation relations $\left[\hat{\phi}(\mathbf{x}, t), \hat{\phi}^{\dagger}\left(\mathbf{x}^{\prime}, t^{\prime}\right)\right]=-\imath \hbar \delta(\mathbf{x}-$ $\left.\mathbf{x}^{\prime}\right) \delta\left(t-t^{\prime}\right)$ and $\left[\hat{\phi}(\mathbf{x}, t), \hat{\phi}\left(\mathbf{x}^{\prime}, t^{\prime}\right)\right]=0$. We defined $\hat{\rho}(\mathbf{x}, t)=$ $\hat{\phi}^{\dagger}(\mathbf{x}, t) \hat{\phi}(\mathbf{x}, t)$ and allowed for an explicit time dependence of the interaction Hamiltonian. Most of the interesting information about the system such as density, spectra, response functions, can be extracted from the knowledge of the single-particle Green's function defined as $G\left(1 ; 1^{\prime}\right)=$ $-\imath\left\langle\mathcal{T}_{\gamma} \hat{\phi}(1) \hat{\phi}^{\dagger}\left(1^{\prime}\right)\right\rangle$, where $\gamma$ is the (properly chosen) Keldysh contour ${ }^{18}$, $20, \mathcal{T}_{\gamma}$ is the time ordering over $\gamma$ and we used the standard notation for variables, namely $1=\left\{\mathbf{x}_{1}, z_{1}\right\}$ and $1^{\prime}=\left\{\mathbf{x}_{1}^{\prime}, z_{1}^{\prime}\right\}, z$ being the complex time variable on the contour. By properly choosing the contour $\gamma$ different approaches can be recovered ${ }^{20}$ : for instance by choosing the contour to run only along the imaginary axis the Matsubara approach to study thermodynamic properties of interacting systems is obtained. We will focus on a contour which goes around the real line running from $z_{0}^{+}=t_{0}+\imath \epsilon$ to $z_{0}^{-}=t_{0}-\imath \epsilon$ $(\epsilon>0)$ crossing the real line at $z_{f}=t_{f}$, namely the maximum evolution time.

As clearly described in Ref. ${ }^{21}$, in order to find the Green's function one can resort to two different, but equivalent approaches. The first uses the Heisenberg equations for the field operators to find an equation of motion for the Green's function. This method results in a hierarchy of equations, the Martin-Swinger hierarchy, in which Green's function of different orders are linked to each other. In our case (two body interaction Hamiltonian) the hierarchy links the time derivative of the $n$-particle Green's function to the $n+1$-particle Green's function. So that the single-particle Green's functions is related to the two-particle one. The second approach is based on the Gell-Mann Low theorem, allowing the cancellation of disconnected diagrams (linked cluster expansion), in order to calculate the Green's function perturbatively by including the wanted set of diagrams whose choice depends upon the physics of the system at hand. This technique is particularly powerful because it allows us to take into account the chosen diagrams to all order in the coupling constant, thus representing an evolution of simple perturbative approach. Here we will follow this second approach.

In this case it is possible to derive a closed set of equations, the Hedin's equations, whose (iterative) solution gives the in- teracting single-particle Green's function:

$$
\begin{aligned}
& G\left(1 ; 1^{\prime}\right)=G_{0}\left(1 ; 1^{\prime}\right)+\int d \overline{1} d \overline{2} G_{0}(1 ; \overline{1}) \Sigma(\overline{1} ; \overline{2}) G\left(\overline{2} ; 1^{\prime}\right)(2) \\
& \Sigma(1 ; 2)=\Sigma_{H}[G, u](1 ; 2)+\Sigma_{e x}[G, W](1 ; 2) \\
& P(1 ; 2)=\imath \int d \overline{1} d \overline{2} G(1 ; \overline{1}) G\left(\overline{2} ; 1^{+}\right) \Lambda(\overline{1} ; \overline{2} ; 2) \\
& W(1 ; 2)=u(1 ; 2)+\int d \overline{1} d \overline{2} u(1 ; \overline{1}) P(\overline{1} ; \overline{2}) W(\overline{2} ; 2) \\
& \Lambda(1,3 ; 2)=\delta\left(1,3^{+}\right) \delta(2,3) \\
& +\imath \int d \overline{1} d \overline{2} d \overline{3} d \overline{4} \frac{\delta \Sigma_{e x}[G, W](1,3)}{\delta G(\overline{1} ; \overline{2})} G(\overline{1} ; \overline{3}) G(\overline{4} ; \overline{2}) \Lambda(\overline{3}, \overline{4} ; 2)
\end{aligned}
$$

where $u(1 ; 2)=u\left(\left|\mathbf{x}_{1}-\mathbf{x}_{2}\right|, z_{1}\right) \delta_{\gamma}\left(z_{1}-z_{2}\right)$. These equations are: the Dyson equation for the single particle Green's function; the equation for the self-energy where $\Sigma_{H}$ and $\Sigma_{e x}$ are the Hartree and exchange self-energy respectively; the equation for the polarization; the equation for the dressed interaction; the equation for the vertex function. All these equations have to be solved iteratively with properly chosen seeds for the iterative scheme; for instance the initial singleparticle Green's function is often chosen to be either the noninteracting one or the self-consistent Hartree Green's function. Nevertheless in trying to solve Hedin's equations iteratively different problems arise. The most difficult to overcome is related to the fact that the equation for the vertex function contains a functional derivative of the (exchange) self-energy with respect to the interacting single particle Green's function. This makes the numerical implementation particularly difficult. Schindlmayr and Godby ${ }^{22}$ have obtained an analytical expression for the vertex function after the first iteration step for the Fermi-Hubbard model. Nevertheless Hedin's approach in its generality still suffers from this problem and in most cases of interest implementing such a scheme is so complicated that other options are usually preferred.

Recently Starke and Kresse used a slightly different point of view in the derivation of a self-consistent set of equations ${ }^{21}$. After observing that, as mentioned above, the single-particle Green's function for a two-body interaction Hamiltonian, is related to the two particle one, they derived a set of two equations which is equivalent to Hedin's equations:

$$
\begin{aligned}
& G\left(1 ; 1^{\prime}\right)=G_{0}\left(1 ; 1^{\prime}\right) \\
& +\imath \int d \overline{1} d \overline{2} G_{0}(1 ; \overline{1}) u(\overline{1} ; \overline{2}) L\left(\overline{2}, \overline{2} ; \overline{1}, 1^{\prime}\right) \\
& L\left(1,2 ; 1^{\prime}, 2^{\prime}\right)=L_{0}\left(1,2 ; 1^{\prime}, 2^{\prime}\right) \\
& +\imath \int d \overline{1} d \overline{2} d \overline{3} d \overline{4} L_{0}\left(1, \overline{1} ; 1^{\prime}, \overline{2}\right) I(\overline{2}, \overline{3} ; \overline{1}, \overline{4}) L\left(\overline{4}, 2 ; \overline{3}, 2^{\prime}\right)
\end{aligned}
$$

where $L\left(1,2 ; 1^{\prime}, 2^{\prime}\right)=G_{2}\left(1,2 ; 1^{\prime}, 2^{\prime}\right)-G\left(1 ; 1^{\prime}\right) G\left(2 ; 2^{\prime}\right)$ with $G_{2}$ being the interacting two-particle Green's function. The two equations are: 1) an equation for the interacting singleparticle Green's function (analogous to the Dyson equation, Eq. 3); 2) the Bethe-Salpeter equation for the exchange part of the interacting two-particle Green's functions. This set of equations does not rely on any functional derivative, but it fixes a priori the functional form of the kernel $I$ of the Bethe- 
Salpeter equation, namely the two-particle scattering amplitude, which on the other hand can be upgraded at every iteration depending on the choice of both $I$ and $L_{0}$ 21 . The latter is in general a functional of the interacting single particle Green's function as well. Depending on the choice of $I$ and $L_{0}$ it is possible to obtain different schemes, including the most used in literature such as the Hartree-fock approximation, the Random-Phase approximation, the GW-approximation, the ladder approximation and the $\mathrm{W}$-approximation (see Table I in Ref ${ }^{21}$ for the hierarchy of approximation for different choices of the kernel $I$ ).

In the following we will introduce our approach closely related to the one derived by Starke and Kresse to find selfconsistently the one- and two-particle Green's functions for the weakly interacting Bose-Hubbard model.

\section{WEAKLY INTERACTING BOSE-HUBBARD MODEL}

The Bose Hubbard model (BHM) is obtained as the tightbinding limit of the Hamiltonian in Eq, 1 where the potential is chosen to have several local minima. In this limit we can choose a basis of wavefunctions localized around the local minima of the potential and write the field operator as $\hat{\phi}(\mathbf{x}, t)=\sum_{i} w_{i}(\mathbf{x}) \hat{b}_{i}(t)$. The Hamiltonian thus reads

$$
\begin{aligned}
\hat{H} & =\hat{H}_{0}+\hat{V}(t) \\
\hat{H}_{0} & =\sum_{i} \epsilon_{i} \hat{b}_{i}^{\dagger} \hat{b}_{i}-\sum_{<i, j>} \frac{J}{2}\left(\hat{b}_{i}^{\dagger} \hat{b}_{j}+\text { h.c. }\right) \\
\hat{V}(t) & =\frac{U(t)}{2} \sum_{i} \hat{b}_{i}^{\dagger} \hat{b}_{i}^{\dagger} \hat{b}_{i} \hat{b}_{i},
\end{aligned}
$$

The operators $\hat{b}_{i}$ and $\hat{b}_{i}^{\dagger}$ obey bosonic commutation relations: $\left[\hat{b}_{i}, \hat{b}_{j}^{\dagger}\right]=\delta_{i, j}$ and $\left[\hat{b}_{i}, \hat{b}_{j}\right]=0$. To fix the ideas we will think of interacting bosonic atoms in an optical lattice for which the $\mathrm{BHM}$ has been proven to give a very good description 13 . In what follows we shall consider the weakly interacting limit $U(t)<J, \forall t$ and resort to a perturbative expansion of the single particle interacting Green's functions. The latter in the interaction picture reads

$$
G\left(1,1^{\prime}\right)=-\imath \frac{\left\langle\mathcal{T}_{\gamma}\left[e^{-\imath \int_{\gamma} d z \hat{V}_{I}(z)} \hat{b}(1) \hat{b}^{\dagger}\left(1^{\prime}\right)\right]\right\rangle}{\left\langle\mathcal{T}_{\gamma}\left[e^{-\imath \int_{\gamma} d z \hat{V}_{I}(z)}\right]\right\rangle}
$$

where $\hat{V}_{I}(z)=U(z) / 2 \sum_{i} \hat{b}_{i}^{\dagger}\left(z^{+}\right) \hat{b}_{i}^{\dagger}\left(z^{+}\right) \hat{b}_{i}(z) \hat{b}_{i}(z)$ is the interaction Hamiltonian in the interaction picture and the multiindex is now $1=\left\{i_{1}, z_{1}\right\}, i_{1}$ being the site index and $z_{1}$ the complex time on the contour. In order to calculate $G\left(1,1^{\prime}\right)$ we resort to its expansion in terms of the non-interacting Green's functions $g\left(1,1^{\prime}\right)$. In particular we use the so called ladder approximation, which amounts to calculate the self-energy entering the Dyson equation by means of a two-particle scattering amplitude which in turn is calculated from the BetheSalpeter equation. For the interaction term as in Eq 11 ,
$G\left(1 ; 1^{\prime}\right)$ can be approximated to second order by expanding the evolution operator. Assuming the initial state is such that the non-interacting $n$-particle Green's functions can be written as a permanent of the non-interacting single-particle ones the denominator in Eq. 12 cancles all disconnected diagrams in the expansion thus leaving only connected ones 20 .

In order to construct our iterative method let us look at the first and second order (connected) contributions which are given by:

$$
\begin{aligned}
G_{c}^{(1)}\left(1 ; 1^{\prime}\right)= & 2 \imath \int_{\gamma} d \overline{1} U\left(\bar{z}_{1}\right) g\left(1 ; \overline{1}^{+}\right) g\left(\overline{1} ; \overline{1}^{+}\right) g\left(\overline{1} ; 1^{\prime}\right)(13) \\
G_{c}^{(2)}\left(1 ; 1^{\prime}\right)= & 2 \imath^{2} \int_{\gamma} d \overline{1} d \overline{2} U\left(\bar{z}_{1}\right) U\left(\bar{z}_{2}\right) \\
& g\left(1 ; \overline{1}^{+}\right) g\left(\overline{1} ; \overline{2}^{+}\right) g\left(\overline{2} ; \overline{1}^{+}\right) g\left(\overline{1} ; \overline{2}^{+}\right) g\left(\overline{2} ; 1^{\prime}\right)
\end{aligned}
$$

In the second order we considered only the two-particle irreducible contributions, i.e. those with no self-energy insertions, the reason will become clear in the following. The factor two comes from the fact that direct and exchange integrals give the same contribution due to the on-site nature of the interaction and to the lack of any other label but the site index of the bosonic operators.

The above terms can be recovered from the Dyson equation for the interacting single-particle Green's function with the definition of a self-energy. We are going to define it by means of a vertex function which is in turn recovered from the Bethe-Salpeter equation whose kernel we chose to be the bare interaction:

$$
\begin{aligned}
& K\left(1^{\prime}, 2^{\prime} ; 1,2\right)=V\left(1^{\prime}, 2^{\prime} ; 1,2\right) \\
& +\imath \int d \overline{1} d \overline{2} d \overline{3} d \overline{4} V\left(1^{\prime}, 2^{\prime} ; \overline{1}, \overline{2}\right) g(\overline{1} ; \overline{3}) g(\overline{2} ; \overline{4}) K(\overline{3}, \overline{4} ; 1,2)
\end{aligned}
$$

where in our case $V\left(1^{\prime}, 2^{\prime} ; 1,2\right)=U\left(z_{1}\right) \delta(2-1) \delta\left(1^{\prime}-\right.$ $\left.1^{+}\right) \delta\left(2^{\prime}-2^{+}\right)$and $\delta\left(1^{\prime}-1^{+}\right)=\delta_{i_{1}^{\prime} i_{1}} \delta_{\gamma}\left(z_{1}^{\prime}-z_{1}^{+}\right)$and we assumed an interaction Hamiltonian of the form $V_{I}=$ $V\left(1^{\prime}, 2^{\prime} ; 1,2\right)=\hat{b}^{\dagger}\left(1^{\prime}\right) \hat{b}^{\dagger}\left(2^{\prime}\right) \hat{b}(1) \hat{b}(2)$. We then define the self-energy as

$$
\Sigma\left(1^{\prime} ; 1\right)=\imath \int d \overline{1} d \overline{2}\left(K\left(1^{\prime}, \overline{2}^{\prime} ; 1, \overline{2}\right)+K\left(1^{\prime}, \overline{2}^{\prime} ; \overline{2}, 1\right)\right) g\left(\overline{2} ; \overline{2}^{\prime}\right)
$$

This is known as the ladder approximation from the form of Feynman diagrams at different orders for the two-particle scattering amplitude $K$. In the case of interacting electrons the ladder approximation is associated to the low density limit and it is physically justified because the contribution of particlehole like excitation is of higher order (in the interaction) with respect to particle-particle scattering in this limit23/24. On the other hand it can also be used to study dilute bosonic gases and their thermodynamical properties 24 .

The definition of a self-energy allows us to write the Dyson equation for the interacting single-particle Green's function:

$$
G\left(1 ; 1^{\prime}\right)=g\left(1 ; 1^{\prime}\right)+\int d \overline{1} d \overline{1}^{\prime} g\left(1 ; \overline{1^{\prime}}\right) \Sigma\left(\overline{1}^{\prime}, \overline{1}\right) G\left(\overline{1} ; 1^{\prime}\right)
$$




\section{SELF-CONSISTENT SOLUTION AND ITS NUMERICAL IMPLEMENTATION}

We have seen that through the definition of a (proper) self-energy it is possible to write a Dyson equation for the interacting single-particle Green's function. Nevertheless the self-energy defined above does not allow to recover many important contributions, in particular those which are one-interaction line reducible. This is because the two-particle scattering amplitude $K$, and therefore the self-energy $\Sigma$ derived from it, does not contain such diagrams by construction. For instance, the following second order contribution cannot be obtained from the Dyson equation with the self-energy defined in Eq. (16), $\imath^{2} \int d \overline{1} d \overline{2} U\left(\bar{z}_{1}\right) U\left(\bar{z}_{2}\right) g(1 ; \overline{1}) g\left(\overline{1} ; \overline{2}^{+}\right) g\left(\overline{2} ; \overline{2}^{+}\right) g\left(\overline{2} ; \overline{1}^{+}\right) g\left(\overline{1} ; 1^{\prime}\right)$. The above second order term can be accounted for by using the Hartree Green's function in the Dyson equation instead of the non-interacting one. Nevertheless there are higher order contributions which cannot be derived by means of the Hartree propagator and the ladder approximation and they would require more complex self-energies to be defined. To get around this problem we resort to iterations by means of the following iterative scheme:

$$
\begin{aligned}
K_{i_{1} j_{1} i_{2} j_{2}}^{(n)}\left(z_{1} ; z_{2}\right) & =V_{i_{1} j_{1} i_{2} j_{2}}\left(z_{1} ; z_{2}\right) \\
& +\imath \sum_{\bar{i}_{1}, \bar{j}_{1}} \int_{\gamma} d \bar{z}_{1} d \bar{z}_{2} V_{i_{1} j_{1} \bar{i}_{2} \bar{j}_{2}}\left(z_{1} ; \bar{z}_{2}\right) G_{\bar{i}_{2} \bar{i}_{1}}^{(n-1)}\left(\bar{z}_{2} ; \bar{z}_{1}\right) G_{\bar{j}_{2} \bar{j}_{1}}^{(n-1)}\left(\bar{z}_{2} ; \bar{z}_{1}\right) K_{\bar{i}_{1} \bar{j}_{1} i_{2} j_{2}}^{(n)}\left(\bar{z}_{1} ; z_{2}\right) \\
\Sigma_{i_{1} i_{2}}^{(n)}\left(z_{1} ; z_{2}\right) & =\imath \sum_{\bar{i}_{1}, \bar{i}_{2}} K_{i_{1} \bar{i}_{1} i_{2} \bar{i}_{2}}^{(n)}\left(z_{1} ; z_{2}\right) G_{\bar{i}_{2} \bar{i}_{1}}^{(n-1)}\left(z_{2} ; z_{1}\right)+\imath \sum_{\bar{i}_{1}, \bar{i}_{2}} K_{i_{1} \bar{i}_{1} \bar{i}_{2} i_{2}}^{(n)}\left(z_{1} ; z_{2}\right) G_{\bar{i}_{2} \bar{i}_{1}}^{(n-1)}\left(z_{2} ; z_{1}\right) \\
G_{i_{1} i_{2}}^{(n)}\left(z_{1} ; z_{2}\right) & =G_{i_{1} i_{2}}^{(0)}\left(z_{1} ; z_{2}\right)+\sum_{\bar{i}_{1}, \bar{i}_{2}} \int_{\gamma} d \bar{z}_{1} d \bar{z}_{2} G_{i_{1} \bar{i}_{1}}^{(0)}\left(z_{1} ; \bar{z}_{1}\right) \Sigma_{\bar{i}_{1} \bar{i}_{2}}^{(n)}\left(\bar{z}_{1} ; \bar{z}_{2}\right) G_{\bar{i}_{2} i_{2}}^{(n+1)}\left(\bar{z}_{2} ; z_{2}\right)
\end{aligned}
$$

with initial seed $G_{i_{1} i_{2}}^{(0)}\left(z_{1} z_{2}\right)=g_{i_{1} i_{2}}\left(z_{1} z_{2}\right)$ being the noninteracting Green's functions and $n$ being the iteration step. In the above equations we split the time and the site indeces, which will turn useful when rewriting the equation for the different components on the contour (see Appendix A). This is nothing but a subset of the most general system of equation, namely the Hedin's equations. It is possible to show that the above set of equations is equivalent to the one in Eqs. (8) with the choice $I(1,2 ; 3,4)=V(1,2 ; 3,4)+V(1,2 ; 4,3)$ and $L_{0}(1,2 ; 3,4)=G(1 ; 3) G(2 ; 4)^{21}$. The difference between our approach and the one presented in Ref ${ }^{21}$ relies on the fact that rather than working with the two-particle Green's functions (namely $L$ ) we use the two-particle scattering amplitude $(K)$. The latter is a tensor which is partially diagonal (Appendix Ap and can thus be implemented as a matrix in the numerical simulations. The two particle Green's function requires four (spatial) indexes in general and it would be more costly in terms of computational resourses. It is important to notice that the self-consistent ladder approximation is a conservative scheme in the sense of Kadanoff and Baym 25 .

In order to make the above system of equations suitable for numerical implementation, each equation has to be decomposed into the corresponding equations with real time arguments which can be done by means of Langreth theorem. The result is the set of equations presented in Appendix A The numerical implementation is then performed by means of two Fortran 90 codes, the first of which solves the non-interacting problem relative to $\hat{H}_{0}$ and passes the non-interacting Green's functions $g$ to the second code which then implements the above iterative scheme. The first code uses OpenMP to speed up the computation of the non-interacting Green's function. The second code uses MPI parallelization by means of the ScaLAPACK libraries on a grid of (usually) 128 processes.

In what follows we will use the iterative scheme in Eqs. (18)- 20 to study the dynamics of one- and twodimensional Bose-Hubbard model following the switching on of the boson-boson interaction $U$. We also compared our approach with results from exact numerical diagonalization (see Appendix B finding good agreement and confirming that we can rely on it for the range of parameter considered in this work.

\section{FIGURES OF MERIT}

To characterize the post-quench dynamics of the BHM we will look at both the expansion of the density of bosons and the spreding of correlations The spreading of bosons over the lattice will be characterized by means of the time-dependent standard deviation of the normalized distribution obtained as $p_{i}(t)=n_{i}(t) / n\left(\sum_{i} p_{i}(t)=1 \quad \forall t\right): \sigma(t)=\left(\overline{i^{2}}(t)-\right.$ $\left.\bar{i}(t)^{2}\right)^{1 / 2}$ where $\bar{i}(t)=\sum_{i} i p_{i}(t)$ and $\overline{i^{2}}(t)=\sum_{i} i^{2} p_{i}(t)$ are the average with respect to the probability distribution $p_{i}(t)$ of the position and its square. The velocity of propagation is given by the time derivative of $v(t)=d \sigma(t) / d t$. We will also look at the momentum distribution defined through the single 
particle density matrix:

$$
n(k)=\frac{\imath}{\sqrt{N}} \sum_{i} e^{-\imath k(i-j)} G_{i, j}^{<}\left(t ; t^{+}\right) .
$$

In order to characterize the spreading of correlations we will look at the evolution of the particle propagator and in particular at its variation $\left|\delta G_{i i_{0}}^{>}(t ; 0)\right|^{2}=\mid G_{i i_{0}}^{>}(t ; 0)-$ $\left.G_{i i_{0}}^{>}(0 ; 0)\right|^{2}$, between the site $i_{0}$ and all other sites as a function of time. $G_{i i_{0}}^{>}(t ; 0)$ gives information on the mobility of a single-particle through the system. In the remainder of the paper we will fix $i_{0}=\lfloor N / 2\rfloor$, other choices changes the results only quantitatively but not qualitatively. The importance of this quantity (and in general of two-times ones) is related to its rôle in the determination of the response of observables of the system to external perturbations, in our case to the quench in the interaction.

\section{QUENCHES IN 1D BOSE-HUBBARD MODEL}

In this section we exploit the presented approach to study the dynamics of a homogeneous one-dimensional BHM following a quench in the boson-boson interaction. In a recent work ${ }^{\sqrt{26}}$ we have shown that the speed of propagation of correlations depends upon the spectrum of the final Hamiltonian. Specifically the speed increases by increasing the bosonboson interaction as suggested by the dispersion relation of excitations in the Bogoliubov theory in the presence of repulsive interaction. Our analysis was limited to small interaction and relied on the Bogoliubov approach, thus giving an effectively non-interacting theory. This is why we could access the ballistic regime only. However it is expected that by increasing the boson-boson interaction the system eventually enters a diffusive regime due to the non-linearity introduced by interactions; this behavior has also been observed experimentally 27 showing that, inside the superfluid phase, as the final interaction strenght is increased, the propagation of particles (density) is diffusive unlike at small interaction where it is ballistic. We now show that the approach introduced above is able to capture such a feature.

\section{A. Zero temperature}

We start by looking at the expansion of the boson density as a function of time and in particular at the speed of propagation $v(t)$ defined above. In Fig 1 we show $v(t)$ for a system with $N=63$ sites and $n=21$ bosons for quenches in the interaction from an initially non-interacting state. We can see that the speed increases in time thus showing the accelerated character of the expansion of the density. This was obviuosly to be expected since the sudden switching-on of the interaction corresponds to a sudden inhomogeneous increase of the energy across the system and in particular it is more concentrated where the density of particles is higher at $t=0$ (the center). This interaction energy is readly converted into kinetic energy resulting in an accelerated expansion of

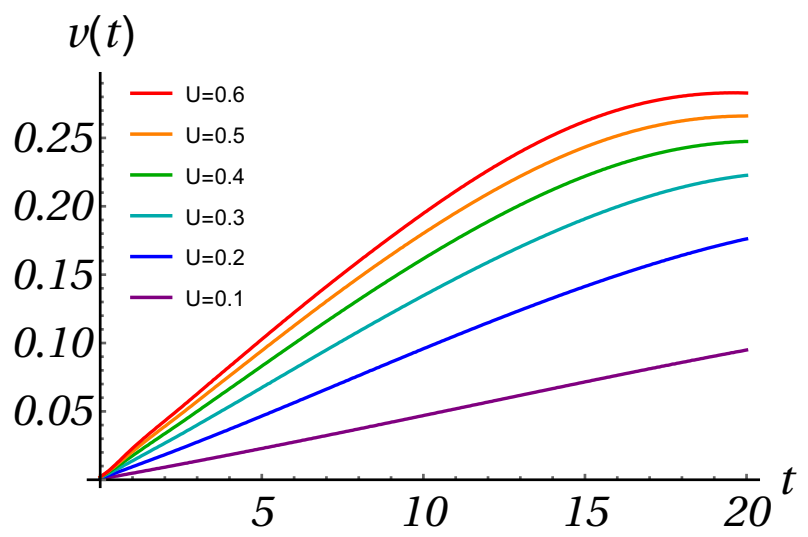

Figure 1: (Color online). Velocity $v(t)$ (see text) for a system with $N=63$ sites and $n=21$ particles initially in the ground state of the (non-interacting) system. Different plots are for different final boson-boson interactions. We set $J=1$.

the density towards the boundaries. We observe that for small interactions the velocity is smaller than for higher ones and this is obviously due to the total initial energy provided during the quench. On the other hand it is interesting to notice that the asymptotic value of the velocity is reached faster at higher interactions (red and orange curves). This can be explained by the fact that although the energy provided to the system increases with the interaction, the rate at which this energy is converted into kinetic energy and distributed across the system is obviously higher for higher interactions due to the increase in the particle-particle scattering processes. We can assume that there exist a transient time, before the system reaches its stationary state, in which the expansion of the density is accelerated up until some time $t^{*}(U)$ after which the expansion slows down and the system starts to equilibrate. This characteristic time corrensponds roughly to the time at which the expansion reaches its maximum speed. From this argument we can also expect that the maximum speed reached at $t=t^{*}(U)$ is such that $v_{M} \propto \sqrt{U}$ because when the number of bosons per site will be $n_{i} \approx 1$ they will become effectively non-interacting and therefore all initial energy, which is $\propto U$, will be converted into kinetic energy. We can check this statement by extrapolating the behavior of the velocity $v(t)$ at longer times. We used a polynomial of sixth order in $t$ to fit $v(t)$ for each value of $U$ and then we extrapolated the maximum of this function in $t$. These values are plotted in Fig 2 . We also show the fitting function of the maxima as a function of $U$ which gives $v_{\infty}(U)=c_{0} U^{\alpha}$ with $c_{0}=(0.368 \pm 0.018)$ and $\alpha=(0.459 \pm 0.047)$ and therefore it is in agreement with our expectation $v_{M}(U) \propto \sqrt{U}$.

In Fig 3 we plot the variation of the particle propagator $\left|\delta G_{i i_{0}}^{>}(t ; 0)\right|^{2}$ between the site $i_{0}$ and all other sites as a function of time for the same system's parameters. The red dashed lines show the light-cone in the non-interacting case. It can be seen that, as $U$ is increased, the particle propagator acquires a diffusive behavior and that interference fringes appear at small $U$ (upper row) due to the coupling of different modes by the interactions; these fringes disappear at higher interactions for 


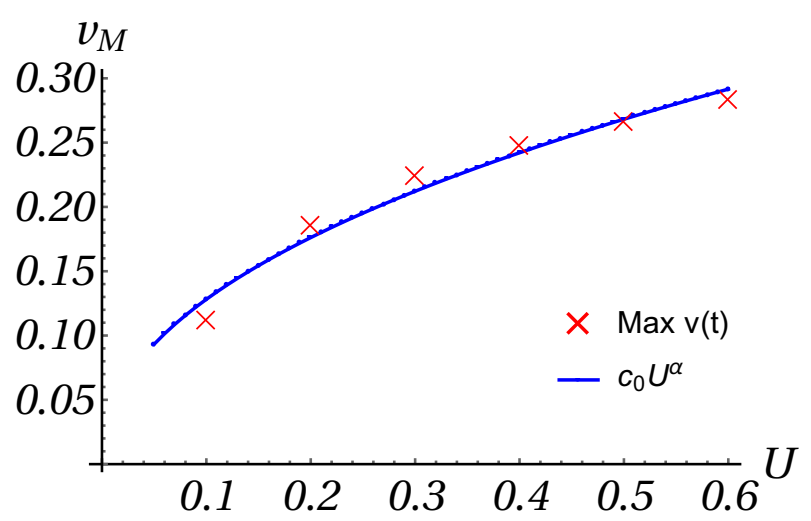

Figure 2: (Color online). Maximum velocity as a function of $U$ obtained from the fit of the curves in Fig 1 with a polynomial of sixth order in $t$. The fitting curve is obtained as $v_{M}(U)=c_{0} U^{\alpha}$ with $c_{0}=(0.368 \pm 0.018)$ and $\alpha=(0.459 \pm 0.047)$.
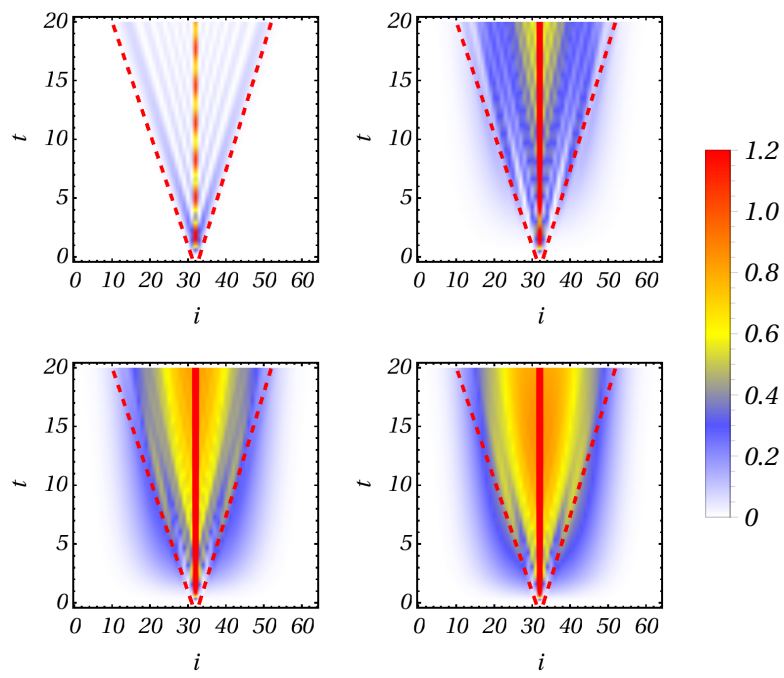

Figure 3: (Color online). Density plot of the variation $\left|\delta G_{i i_{0}}^{>}(t ; 0)\right|^{2}$ of the particle propagator (see text) for a system with $N=63$ sites and $n=21$ particles initially in the ground state of the (noninteracting) system. Different plots are for different final bosonboson interactions: from top left to bottom right $U=0,0.1,0.3,0.5$. Red (dashed) lines are the reference light cone for the non-interacting case $(U=0)$.

long times (bottom row) due to the interaction-induced decoherence in the system.

\section{B. Finite temperature}

It is interesting to understand the effect of the initial temperature on the post-quench dynamics of the system, particularly because the study of many-body systems starting from an initial Gibbs state has attracted recently a lot of interest in the context of quantum thermodynamics. To study the effect of temperature on the post-quench dynamics of the system we consider an initial Gibbs state of the form
$\hat{\rho}=e^{-\beta\left(\hat{H}_{0}-\mu \hat{N}\right)} / \operatorname{Tr}\left(e^{-\beta\left(\hat{H}_{0}-\mu \hat{N}\right)}\right)$ where $\hat{N}=\sum_{i} \hat{b}_{i}^{\dagger} \hat{b}_{i}$ is the total-number operator.

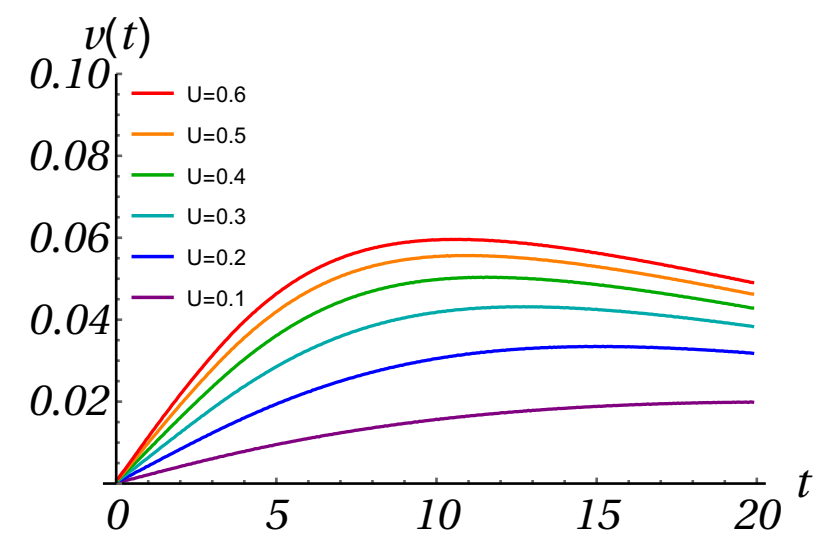

Figure 4: (Color online). Velocity $v(t)$ (see text) for a system with $N=63$ sites, $n=21$ particles. The initial state is assumed to be prepared with an initial inverse temperature $\beta=1$. Different curves are for different final interaction strengths.

In Fig 4 we plot the velocity of expansion of the density $v(t)$ for the case $\beta=T^{-1}=10$ and for a system of $N=63$ sites and an average number of bosons $\langle\hat{N}\rangle=21$. Different curves are for different final interactions $U$. Comparing corresponding curves with the ones in Fig 1 we see that the effect of temperature is to decrease the expansion velocity. On the other hand the argument used to predict the behavior of the maximum expansion velocity with respect to the interaction strength $U$ is still valid as we can observe by fitting the maximum value for each $U$ with a power law $v_{M}(U)=c_{0} U^{\alpha}$ finding $\alpha=(0.566 \pm 0.038)$ (see Fig 5 ).

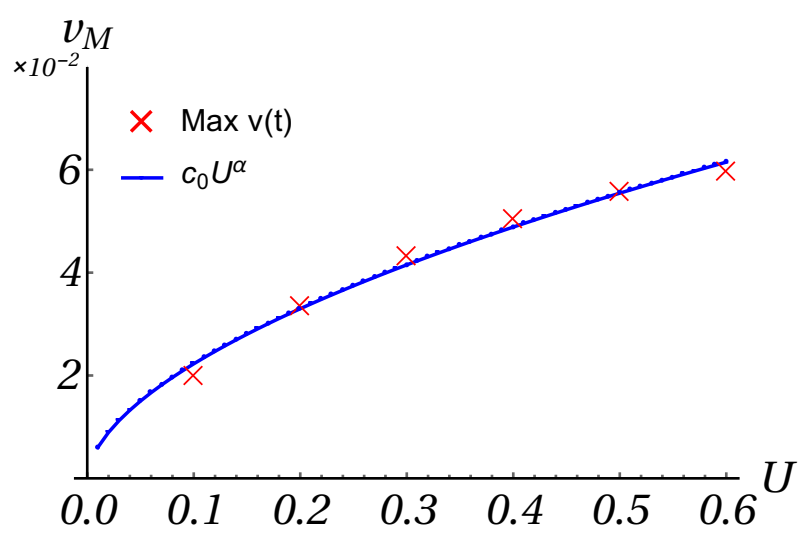

Figure 5: (Color online). Maximum velocity as a function of $U$ obtained from the fit of the curves in Fig 4 with a polynomial of sixth order. The fitting curve is obtained as $v_{M}(U)=c_{0} U^{\alpha}$ with $c_{0}=(0.082 \pm 0.003)$ and $\alpha=(0.566 \pm 0.038)$.

For fixed final interaction $U$ and for different initial inverse temperatures $\beta$ we can see from Fig 6 that an increase in temperature results in a suppression of the expansion of the system. 


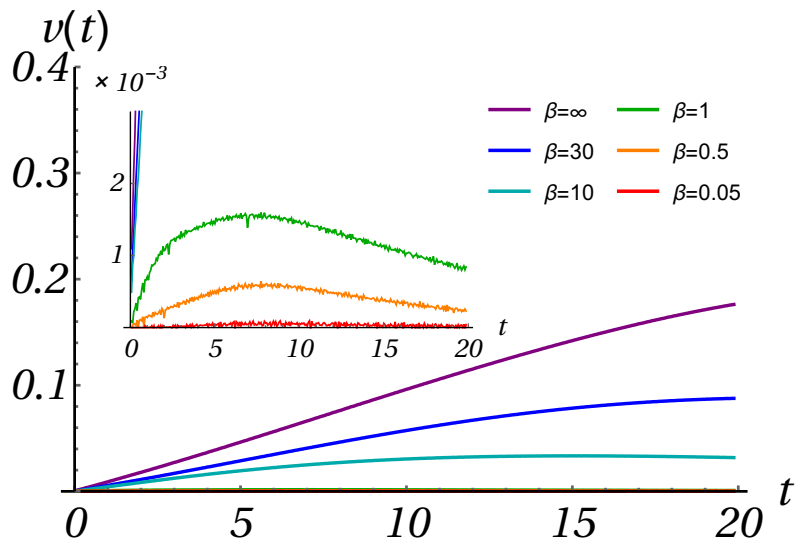

Figure 6: (Color online). Velocity $v(t)$ (see text) for a system with $N=63$ sites, $n=21$ particles and final interaction $U=0.2$. Different curves are for different initial inverse temperature $\beta=$ $\infty, 30,10,1,0.5,0.05$.

This behavior can be understood qualitatively by the fact that as temperature increases, particles in the initial state tend to occupy more energy eigenstates of the non-interacting Hamiltonian and therefore the systems becomes effectively more dilute in the energy eigenstates. Therefore as the interaction is switched on the number of particles which are coupled decreases with the initial temperature. From a formal point of view the interaction Hamiltonian in the momentum basis (assuming periodic boundary conditions) reads: $\hat{V}(t)=\frac{U(t)}{2 L} \sum_{k, p, q} \hat{\tilde{b}}_{p+q}^{\dagger} \hat{\tilde{b}}_{k-q}^{\dagger} \hat{\tilde{b}}_{p} \hat{\tilde{b}}_{k}=\frac{U(t)}{2 L} \sum_{k, p} \hat{\tilde{b}}_{p}^{\dagger} \tilde{\tilde{b}}_{k}^{\dagger} \hat{\tilde{b}}_{p} \hat{\tilde{b}}_{k}+$ $\frac{U(t)}{2 L} \sum_{k, p, q \neq 0} \cdots$. In a dilute system as the one considered here the leading contribution is the first one ( $\mathrm{q}=0$ ) which contains the product $\hat{\tilde{n}}_{p} \hat{\tilde{n}}_{k}$ of the number operators for the states with momenta $p, k$.

To be more quantitative let us look at the distribution of particles in momentum basis both at $t=0$ (initial state) and at the end of the evolution. Bearing this in mind we see from Fig 7 (left) that at low temperatures the momentum distribution is peaked around $k=0$ and, as expected, most of particles occupy low energy states. On the other hand at high initial temperatures the initial momentum distribution is spread over the whole $k$-space meaning that the system tends to occupy more energy levels as it is natural to expect when temperature is increased. We can see the effect of switching-on the interaction on the momentum distribution in the right panel of Fig 7 It shows that for low-temperature initial states the variation of the number of particles at $k \approx 0$ is nearly three order of magnitude greater than for high-temperature initial states. This means that for the latter case the system is, within a good approximation, basically non-interacting (as also expected by the expression for the interaction Hamiltonian in the momentum basis).

The propagation of correlations is also influenced by the temperature of the initial state. In Fig 8 we show the variation of the particle propagator $\delta G^{>}\left(t, t^{+}\right)$for a system with initial inverse temperature $\beta=10$ for different final interaction strengths $U=0,0.1,0.3,0.5$. Comparing the case $U=0$
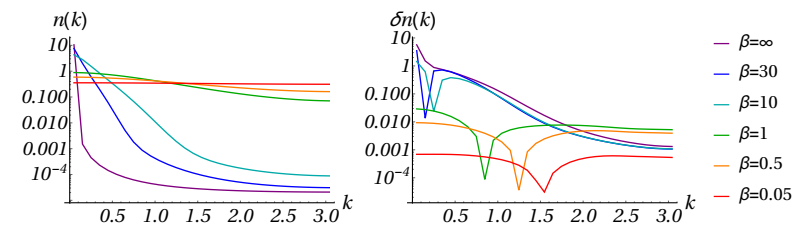

Figure 7: (Color online). Initial momentum distribution $n(k)$ (left) and variation of the momentum distribution $\delta n(k)$ at $\mathrm{t}=20$ for a system with $N=63$ sites, $n=21$ particles and final interaction strength $U=0.2$. Different curves are for different initial temperature $T=\beta^{-1}$ of the initial state.

with the corresponding case at zero temperature (Fig 3 top-left plot) we see that the maximum speed of propagation (given by the slope of the dashed red lines) is the same. This is obviously to be expected because it only depends upon the spectrum which is the same for both cases. By increasing the final interaction strength $U$ we again have a focalization effect and the spreading of correlations slows down as in the case at zero temperature.
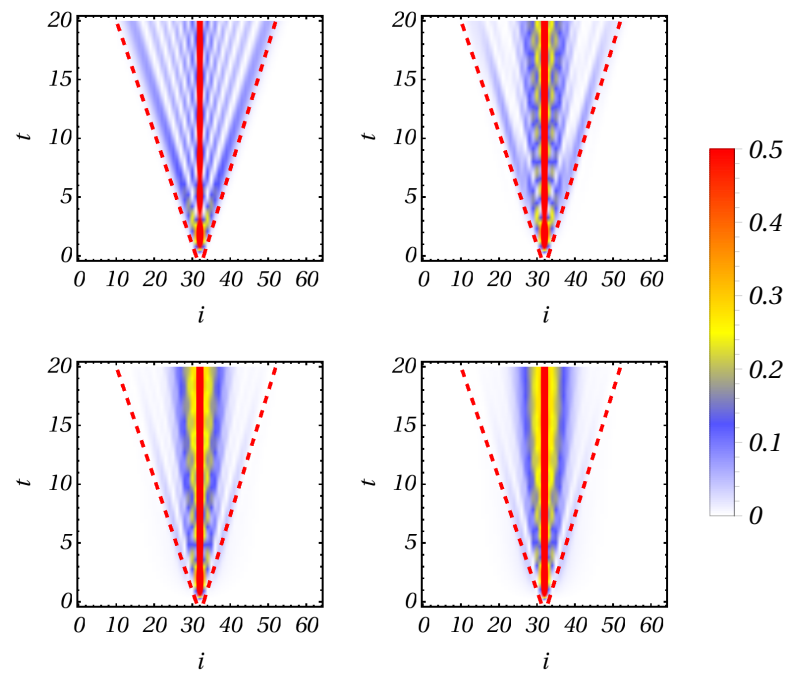

Figure 8: (Color online). Density plot of the variation of the particle propagator (see text) for a system with $N=63$ sites and $n=21$ particles. The initial state is a Gibbs state of the form $\hat{\rho}=$ $e^{-\beta\left(\hat{H}_{0}-\mu \hat{N}\right)} / \operatorname{Tr}\left(e^{-\beta\left(\hat{H}_{0}-\mu \hat{N}\right)}\right)$ with inverse temperature $\beta=1$. The chemical potential $\mu$ is chosen such that the number of particles in the system is $\langle\hat{N}\rangle_{\hat{\rho}}=21$. Different plots refer to different interactions, from top left to bottom right: $U=0,0.1,0.3,0.5$.

On the other hand if we fix the final interaction strength and we look at the particle propagator for different initial temperatures we see that by increasing it the propagation becomes more similar to the non-interacting case. This is clearly shown in Fig 9 and by comparison with the top left figure in Fig. 3 . Therefore we can say that as the initial temperature increases the propagation of correlations becomes ballistic. This behavior can be explained once again by looking at the distribution of particles in the momentum basis: at higher temperatures the effect of interactions is negligible due to the low occupancy of 
each mode.
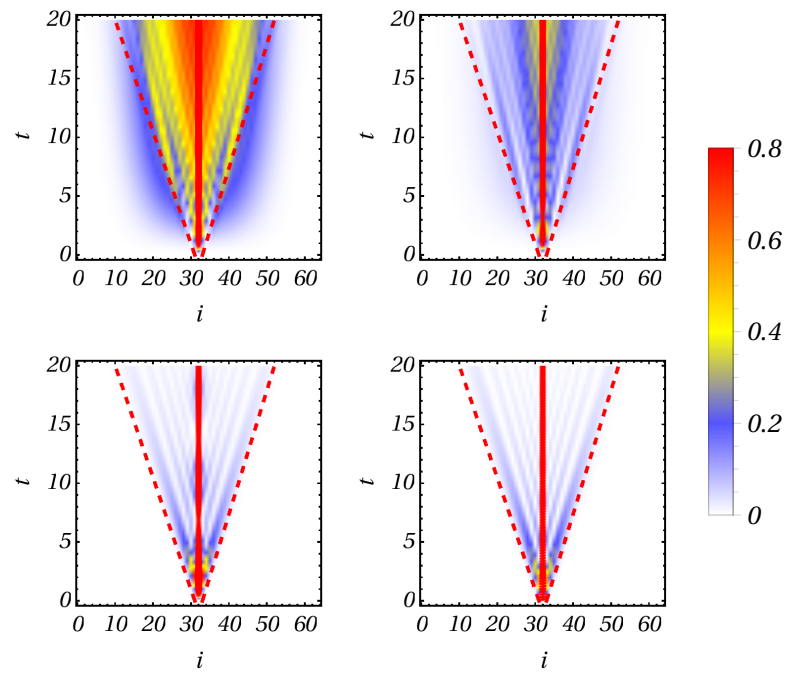

Figure 9: (Color online). Density plot of the variation of the particle propagator (see text) for a system with $N=63$ sites and $n=21$ particles and final interaction $U=0.2$. The initial state is a Gibbs state of the form $\hat{\rho}=e^{-\beta\left(\hat{H}_{0}-\mu \hat{N}\right)} / \operatorname{Tr}\left(e^{-\beta\left(\hat{H}_{0}-\mu \hat{N}\right)}\right)$ with inverse temperature $\beta=1$. The chemical potential $\mu$ is chosen such that the number of particles in the system is $\langle\hat{N}\rangle_{\hat{\rho}}=21$. The different plots refer to different initial inverse temperature, from top left to bottom right: $\beta=\infty, 30,1,0.05$.

\section{QUENCHES IN 2D BOSE-HUBBARD MODEL}

We now move the study of the post quench dynamics of a two-dimensional BHM on a square lattice of size $N_{x} \times N_{y}=$ $21 \times 21$. We will see that qualitatively the results are similar to the one-dimensional case studied in the previous section.

\section{A. Zero Temperature}

It has been showr ${ }^{27}$ that in the case of a two-dimensional gas of bosons on a lattice, the asymptotic velocity decreases by increasing the final boson-boson interaction for quenches from the Mott insulator phase into the superfluid one.

In our case we see from Fig 10 that by increasing the final boson-boson interaction strength the velocity increases during the transient as it is expected from simple energy conservation arguments. In Fig. 10 we plot the velocity related to the $\sigma(t)$ relative to the motion along the $x$-axis only because the system is isotropic and therefore the expansion is the same along any direction. Furthermore, as in the one-dimensional case, we can see from Fig. 11 that the maximum velocity reached has a square root dependence upon the final interaction strength which again confirms the conversion of the initial interaction energy injected into the system by the quench into kinetic energy.

On the other hand in an anisotopic system $\left(J_{y} \neq J_{x}\right)$ the velocity is expected to be different along different directions. In

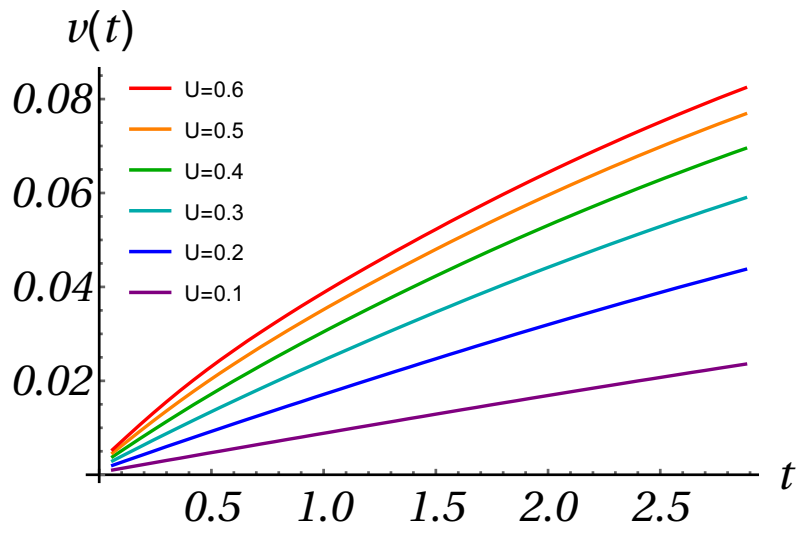

Figure 10: (Color online). Velocity $v(t)$ (see text) for a twodimensional the isotopic system $\left(J_{x}=J_{y}\right)$ with $N_{x} \times N_{y}=21 \times 21$ sites and $n=147$ particles initially in the ground state of the (noninteracting) system. Different plots are for different final bosonboson interactions.

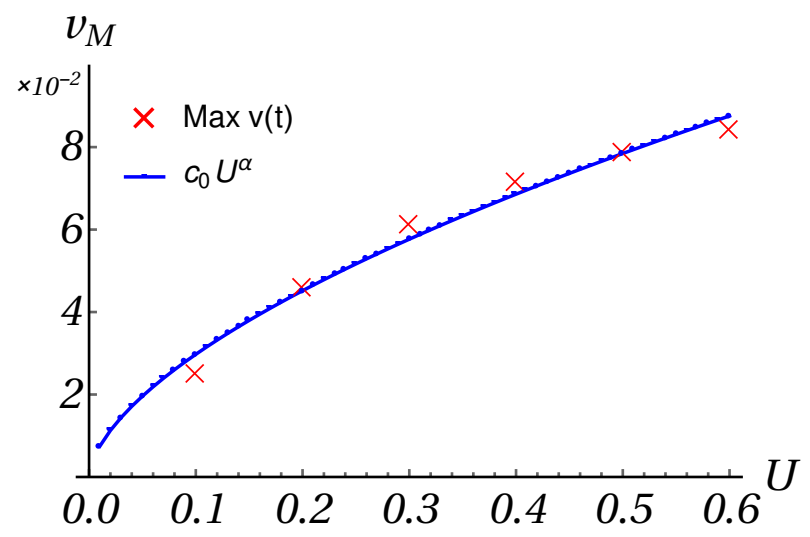

Figure 11: (Color online). Maximum velocity as a function of $U$ obtained from the fit of the curves in Fig 10 with a polynomial of sixth order in $t$. The fitting curve is obtained as $v_{M}(U)=c_{0} U^{\alpha}$ with $c_{0}=(0.119 \pm 0.006)$ and $\alpha=(0.602 \pm 0.055)$.

Fig 12 and Fig 13 we see that the maximum velocity along the $x-$ and $y$-axis respectively is different not only in the value, but also on its dependence upon the tunneling rate along the $y$-axis. In particular by tightening the trap along the $y$-axis the maximum velocity along the $x$-direction is suppressed exponentially with $1 / J_{y}$ whereas the maximum velocity along the $y$-axis has a power law dependence upon $J_{y}$.

It is also interesting to study the rôle of dimensionality in the propagation of correlations, namely the crossover from an isotropic two dimensional lattice $\left(J_{x}=J_{y}\right)$ to an anisotropic one $\left(J_{x}>J_{y}\right)$. In the one-dimensional case we found that the propagation of correlations turns from ballistic to diffusive as the final interaction strength increases. In two dimensions we have a similar behavior as seen in Fig. 14 where we show the variation of the particle propagator $\left|\delta G_{\mathbf{r r}_{0}}^{>}(t ; 0)\right|$, where now $\mathbf{r}=(i, j)$ and $\mathbf{r}_{0}=(\lfloor N / 2\rfloor+1,\lfloor N / 2\rfloor+1)$, for an isotropic system $\left(J_{x}=J_{y}\right)$ with $N_{x} \times N_{y}=21 \times 21$ sites. All plots refer to $t=3$ whereas from top left to bottom right the 


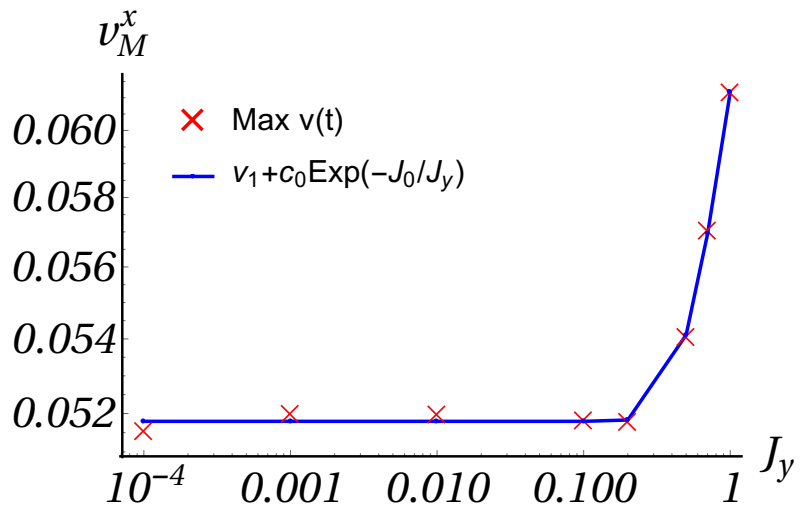

Figure 12: (Color online). Maximum velocity along the $x$-axis as a function of $J_{y}$ for a two-dimensional anisotopic system with $N_{x} \times$ $N_{y}=21 \times 21$ sites and $n=147$ particles initially in the ground state of the (non-interacting) system. The fitting curve is obtained as $v_{M}\left(J_{y}\right)=v_{1}+c_{0} \exp \left(-J_{0} / J_{y}\right)$ with $v_{1}=(0.05179 \pm 0.00007)$, $c_{0}=(0.038 \pm 0.002)$ and $J_{0}=(1.400 \pm 0.065)$.

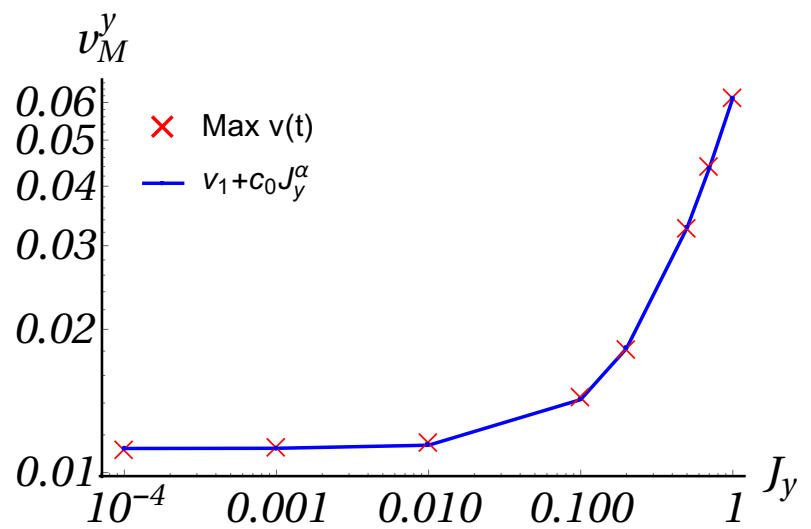

Figure 13: (Color online). Maximum velocity along the $y$-axis as a function of $J_{y}$ for a two-dimensional anisotopic system with $N_{x} \times$ $N_{y}=21 \times 21$ sites and $n=147$ particles initially in the ground state of the (non-interacting) system. The fitting curve is obtained as $v_{M}\left(J_{y}\right)=v_{1}+c_{0} J_{y}^{\alpha}$ with $v_{1}=(0.0112 \pm 0.0001), c_{0}=$ $(0.0500 \pm 0.0002)$ and $\alpha=(1.22 \pm 0.01)$.

interaction strength takes the values $U=0.005,0.1,0.2,0.4$. We can see that at small interactions (top row) a square "wave front" spreads ballistically from the center whereas at higher interactions (bottom row) the propagation becomes diffusive as witnessed by a guassian-like distribution.

In an anisotropic system the spreading of correlations has different behaviors along the two axis as it would have been expected. This can be clearly seen in Fig. 15 where we considered the case $J_{y} / J_{x}=10^{-3}$. At small interaction strengths we can clearly see the difference between the propagation in the two directions whereas as the interaction is increased the propagation tends to become more homogeneous. This can be explained by the following argument: in the limit $J_{y} \rightarrow 0$ the system behaves as weakly coupled one dimensional BoseHubbard models in the $y$-direction. The gas is therefore made of one dimensional "pipes" with lower density than the
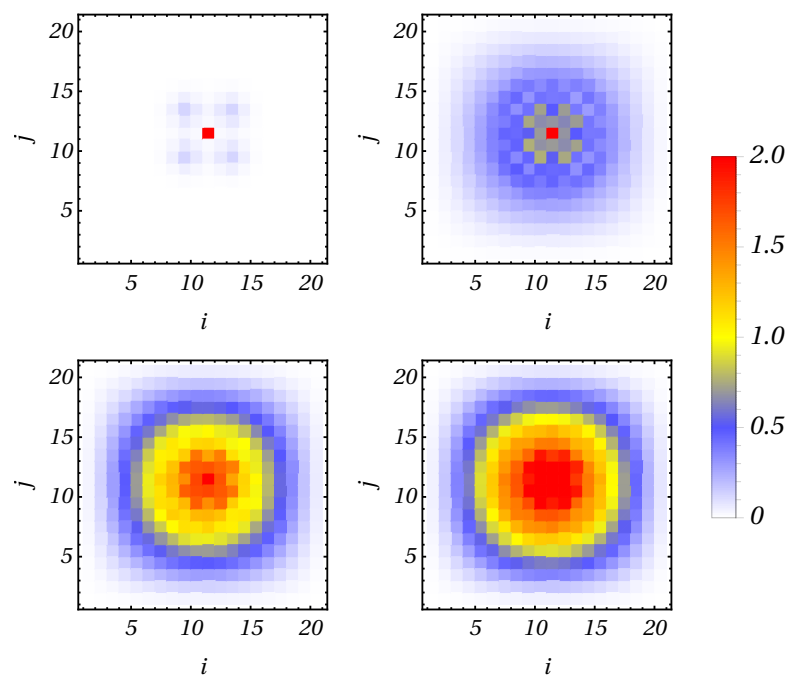

Figure 14: (Color online). Density plot of the variation $\left|\delta G_{i i_{0}}^{>}(t ; 0)\right|^{2}$ of the particle propagator (see text) for a two-dimensional the isotopic system $\left(J_{x}=J_{y}\right)$ with $N_{x} \times N_{y}=21 \times 21$ sites and $n=147$ particles initially in the ground state of the (non-interacting) system. Different plots are for different final boson-boson interactions: from top left to bottom right $U=0,0.1,0.3,0.5$ at $t=3$.
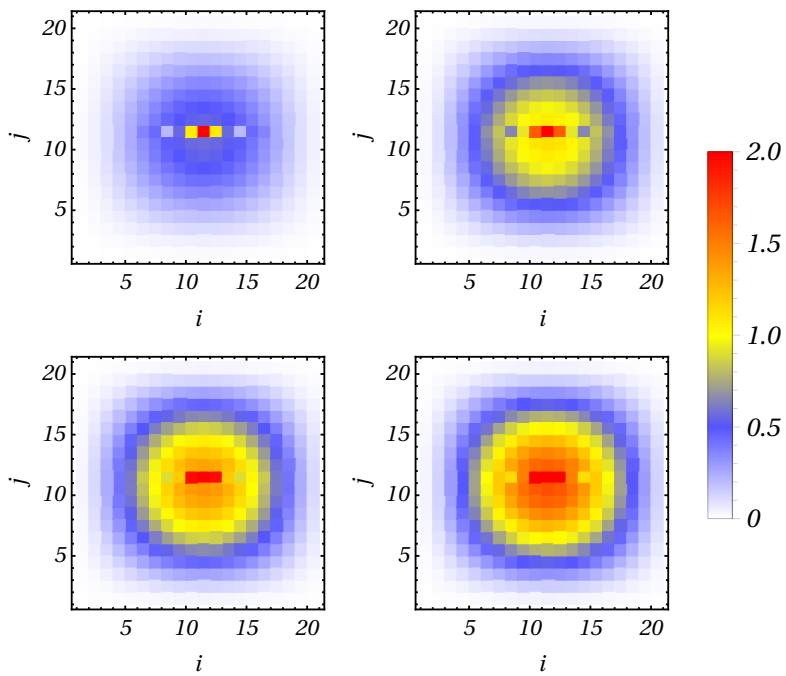

Figure 15: (Color online). Density plot of the variation of the particle propagator (see text) for an anisotropic two-dimensional system with $N_{x} \times N_{y}=21 \times 21$ sites and $n=147$ particles initially in the ground state of the (non-interacting) system. The tunneling rate along the $y$-direction in units of the tunneling rate along the $x$-direction $J_{x}$ is $J_{y}=0.001$ Different density plots correspond to different final interactions (from top left to bottom right) $U=0.1,0.2,0.3,0.4$ at $t=3$.

total one. Since the effect of the interaction depends crucially on the density of the system the expansion velocity decreases with the initial density of the system and therefore also in this case the average velocity tends to decrease in the limit $J_{y} \rightarrow 0$, as shown in Fig. 10, in agreement with recent finding $s^{28}$ obtained by Gutzwiller ansatz. 


\section{B. Finite Temperature}

We have seen in Sec VI that for a 1D BHM the effect of the initial temperature is to slow down the expansion of the density following a quench in the interaction. At very high temperature the expansion is even slower than that in the noninteracting case. In two dimensions we have a similar effect as it can be seen from Fig 16 Similarly to the one dimensional

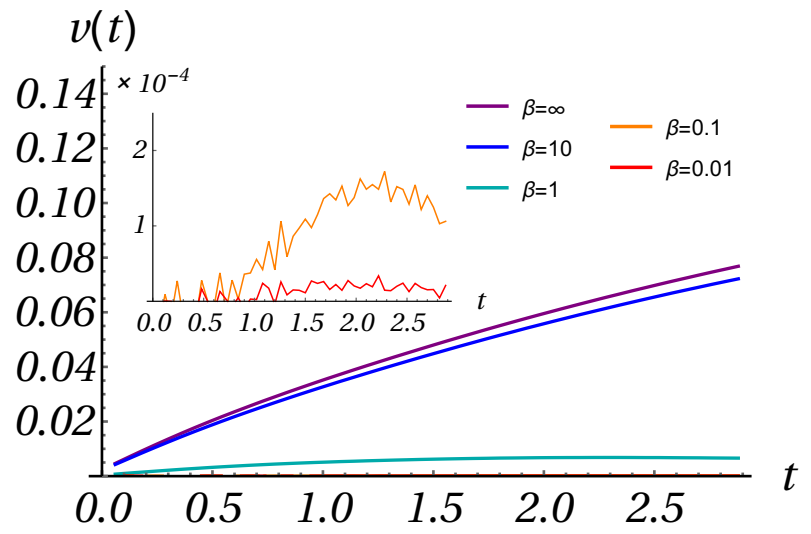

Figure 16: (Color online). Velocity $v(t)$ (see text) along the $\mathrm{x}$ direction for an isotrpic two-dimensional BHM with $N_{x} \times N_{y}=$ $21 \times 21$ sites, $n=147$ particles. Different curves are for different initial temperatures $T=\beta^{-1}$. In the inset we show a zoom in for the curves at high initial temperatures. The final interaction strength is set to $U=0.5$.

case the reason for different behaviors at low and high initial temperature cases resides in the initial occupancy of higher energy levels (left panel in Fig. 17). At fixed average number of particles, the temperature makes the system more dilute in the energy levels therefore reducing the scattering amplitude between different energy eigenstates as witnessed by the difference in the final and initial momentum distribution shown in the right panel of Fig. 17 .
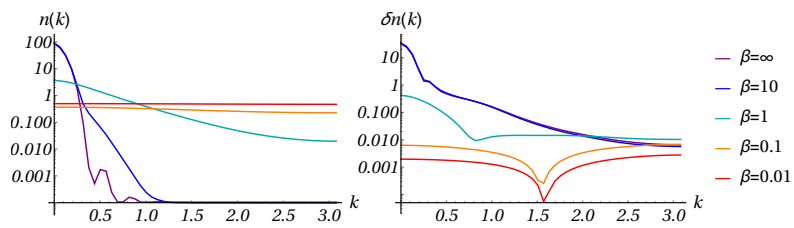

Figure 17: (Color online). Initial momentum distribution $n(k)$ (left panel) and variation of the momentum distribution $\delta n(k)$ at $\mathrm{t}=3$ (right panel) for a system with $N_{x} \times N_{y}=21 \times 21$ sites, $n=147$ particles and final interaction $U=0.5$. We plot a cut of the two dimensional momentum distribution for $k_{x}=k_{y}=k$ where $k_{x}$ and $k_{y}$ are the wavevector in the $x-$ and $y$ - direction respectively. Different curves are for different initial temperature $T=\beta^{-1}$ of the initial state.

In two dimensions the temperature plays a similar rôle as one can see in Fig. 18 where it is shown that by increasing the temperature the propagation of correlations turns from diffu- sive (top row) to ballistic (bottom row).
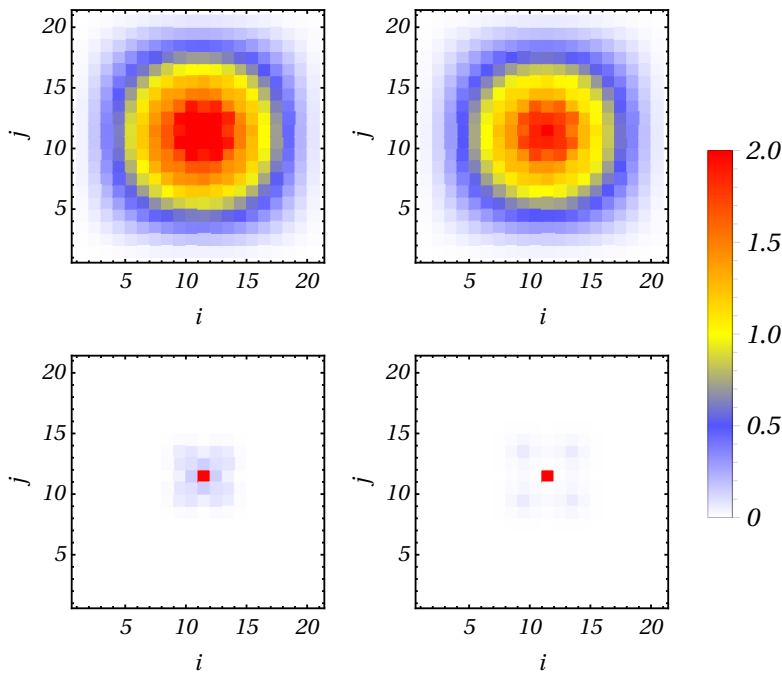

0.5

0

Figure 18: (Color online). Density plot of the variation of the particle propagator (see text) for a isotropic two-dimensional system with $N_{x} \times N_{y}=21 \times 21$ sites,$n=147(n / N=1 / 3)$ particles, and final interaction $U=0.5$. The plots are taken at time $t=3.0$. The initial state is a Gibbs state of the form $\hat{\rho}=$ $e^{-\beta\left(\hat{H}_{0}-\mu \hat{N}\right)} / \operatorname{Tr}\left(e^{-\beta\left(\hat{H}_{0}-\mu \hat{N}\right)}\right)$ with inverse temperature (from top left to bottom right) $\beta=\infty, 10,1,0.1$. The chemical potential $\mu$ is chosen such that the number of particles in the system is $\langle\hat{N}\rangle_{\hat{\rho}}=147$.

\section{CONCLUSIONS}

We have presented an approach for studying the dynamics of weakly interacting Bose-Hubbard model based on the iterative solution of a set of equations for the single and two particle Green's functions in the framework of the non-equilibrium Green's functions (Schwinger-Keldysh formalism). The advantage of such an approach with respect to other widely used techniques such as exact numerical diagonalization and time dependent density matrix renormalization group is that it allows for the calculation of two-time correlation functions for relatively large systems in one and two dimensions. We applied this approach to study global quenches in the interaction parameter for one- and two-dimensional BHM, finding the crossover from ballistic to diffusive regime in the propagation of correlations and the temperature effects in the dynamics. The approach presented here is also suitable for extensions in different directions such as the study of the dynamics from a general interacting initial state by allowing the contour $\gamma$ to have a branch over the imaginary axis $\frac{20}{20}$ the study of strong coupling limi ${ }^{14115}$, the study of transport in the presence of thermal reservoirs ${ }^{3}$. 


\section{ACKNOWLEDGMENTS}

We thank Margherita Marsili for useful discussions and acknowledge financial support from MIUR through FIRB Project No. RBFR12NLNA_002. NLG acknowledges financial support from the EU collaborative project QuProcS (Grant Agreement 641277). We thank the Department of Physics of the Universitá della Calabria for providing access to high performance computational resources through the NEWTON cluster (Progetto PONa3_00370 Materiali, Tecnologie e Ricerca Avanzata - MaTeRiA).

\section{Appendix A: Formal solution for the real time components}

In this section we give the formal solution for the Keldysh components of the interacting single particle Green's functions. We start from the Bethe-Salpeter equation for the kernel $K$. We have seen that the only non-vanishing terms are $K_{i_{1} i_{1} ; i_{2} i_{2}}\left(z_{1} ; z_{2}\right)$ and thus we can write it as the product $K_{i_{1} j_{1} ; i_{2} j_{2}}\left(z_{1} ; z_{2}\right)=k_{i_{1} ; i_{2}}\left(z_{1} ; z_{2}\right) \delta_{i_{1}, j_{1}} \delta_{i_{2}, j_{2}}$ with $k_{i_{1} ; i_{2}}\left(z_{1} ; z_{2}\right)=K_{i_{1} i_{1} ; i_{2} i_{2}}\left(z_{1} ; z_{2}\right)$. We can thus rewrite the Bethe-Salpeter equation as a Fredholm equation::

$$
\begin{aligned}
k_{i_{1} i_{2}}\left(z_{1} ; z_{2}\right)= & U\left(z_{1}\right) \delta_{i_{1} i_{2}} \delta_{\gamma}\left(z_{1}-z_{2}\right) \\
& +\sum_{\bar{i}_{1}} \int_{\gamma} d \bar{z}_{1} A_{i_{1} ; \bar{i}_{1}}\left(z_{1} ; \bar{z}_{1}\right) k_{\bar{i}_{1} i_{2}}\left(\bar{z}_{1} ; z_{2}\right),
\end{aligned}
$$

where we defined the kernel of the integral equation as $A_{i_{1} i_{2}}\left(z_{1} ; z_{2}\right)=\imath U\left(z_{1}\right) g_{i_{1} i_{2}}\left(z_{1} ; z_{2}\right) g_{i_{1} i_{2}}\left(z_{1} ; z_{2}\right)$.

Using Langreth's theorem we can write the equations satisfied by the retarded $(R)$, advanced $(A)$, lesser $(<)$ and greater $(>)$ components:

$$
\begin{aligned}
k^{R / A} & =U \delta+A^{R / A} \circ k^{R / A} \\
k^{\lessgtr} & =A^{R} \circ k^{\lessgtr}+A^{\lessgtr} \circ k^{A},
\end{aligned}
$$

where we dropped the indexes and introduced the symbol $\circ$ which replaces the sums and integrals. The above equations have solutions:

$$
\begin{aligned}
k^{R / A} & =U\left(1-A^{R / A}\right)^{-1} \\
k^{\lessgtr} & =\left(1-A^{R}\right)^{-1} \circ A^{\lessgtr} \circ k^{A},
\end{aligned}
$$

whereas for the components of the self energy we have:

$$
\begin{aligned}
\Sigma_{i_{1} i_{2}}^{\lessgtr}\left(t_{1} ; t_{2}\right) & =2 \imath k_{i_{1} i_{2}}^{\lessgtr}\left(t_{1} ; t_{2}\right) g_{i_{2} i_{1}}^{\gtrless}\left(t_{2} ; t_{1}\right) \\
\Sigma^{R}{ }_{i_{1} i_{2}}\left(t_{1} ; t_{2}\right) & =\Theta\left(t_{1}-t_{2}\right)\left(\Sigma^{>}{ }_{i_{1} i_{2}}\left(t_{1} ; t_{2}\right)-\Sigma^{<}{ }_{i_{1} i_{2}}\left(t_{1} ; t_{2}\right)\right) \\
\Sigma^{A}{ }_{i_{1} i_{2}}\left(t_{1} ; t_{2}\right) & =\Theta\left(t_{2}-t_{1}\right)\left(\Sigma^{<}{ }_{i_{1} i_{2}}\left(t_{1} ; t_{2}\right)-\Sigma^{>}{ }_{i_{1} i_{2}}\left(t_{1} ; t_{2}\right)\right) .
\end{aligned}
$$

It is now easy to find the solution for the different components of the Dyson equation giving the different components of the

interacting single-particle Green's functions:

$$
\begin{aligned}
G^{R / A} & =\left(1-g^{R / A} \circ \Sigma^{R / A}\right)^{-1} \circ g^{R / A} \\
G^{\lessgtr} & =\left(1-g^{R} \circ \Sigma^{R}\right)^{-1} \circ\left(g^{\lessgtr}+g^{R} \circ \Sigma^{\lessgtr} \circ G^{A}+g^{\lessgtr} \circ \Sigma^{A} \circ G^{A}\right) .
\end{aligned}
$$

\section{Appendix B: Comparison with exact solution}

Here we compare the dynamics of a one dimensional BHM as ottained by the self-consistent ladder aproximation with the results obtained by exact diagonalization. Due to the heavy computational requirements of the exact diagonalization, we restrict the comparison to the zero temperature case and small sized systems. Furthermore we will only look at of equal time correlation functions such as numeber of bosons per site and single particle reduced density matrix. The exact diagonalization is done by considering $n$ bosons in $N$ sites allowing for a maximum number of bosons at each site $m_{i}=n$. There- 
fore the exact solution does not suffer from reduction of the whole Hilbert space with $n$ bosons to some of its subspaces (e.g. $m_{i}<n$ ). The price to pay for this is obviously that we cannot study the dynamics of too large systems and/or with too many bosons.

In the main text we studied the post-quench dynamics in homogenous systems with open boundary conditions. Here, in order to compare the dynamics given by our approach to the exact one we will consider both this case (homogeneous) and a one with a slightly richer dynamics. Specifically we will assume the presence of an harmonic trap at the left boundary of the system $\left(\epsilon_{i}=5 \times 10^{-3}(i-2)^{2}\right)$. In this case the ground state of the Hamiltonian shows a distribution around the minima of the potential and, at zero temperature, all bosons initially occupy this state at $t=0^{-}$. The switching on of the (repulsive) boson-boson interaction at $t=0$ will make the cloud expand. Due to the open boundary conditions, all bosons will tend to go towards the right end of the system $(i>2)$ thus developing a non-zero total momentum which comes from the reflection at the left boundary.

In Fig 19 and Fig 20 we show the average number of bosons per site for a sistem with $N=15$ sites and $n=5$ bosons ( $\rho=n / N=1 / 3$ ) for the homogeneous and inhomogeneous case respectively. We compare the exact dynamics (EX) (blue circles), self-consistent ladder approximation (SCL) (red x's) and the self-consistent Hartree-Fock (SCHF) (green crosses). In each figure, plots refer to two different times (top) $t=3$ and (bottom) $t=5$ and for two different values of the interaction: $U=0.3$ (left panel) and $U=0.6$ (right panel). We can see that the SCL approximation captures really well the main features of the exact evolution both qualitatively and quantitatively as opposite to the SFHF which fails to describe both short and long time dynamics (here long times is with respect to the perturbation reaching the boundaries). Moreover we see that even at higher interactions the SCL still gives accurate results. This might seem surprising at first sight but it is not, the ladder approximation, by construction, includes contribution from multiple particle-particle scattering. Together with the self-consistent approach this allows including these diagrams to all orders in $U$. The only limitation comes therefore from physical processes which are not encompassed by the ladder expansion.

We can also show that, in the regimes considered here (dilute gases and/or weak interactions), the discrepancy between the EX and the SCL reduces with the system size. In Fig 21 we plot the maximum deviation over the time interval at each site $\Delta_{i}=\max _{t}\left|n_{i}^{E X}(t)-n_{i}^{S C L}(t)\right|$ for three different system sizes: (blue) $N=9$, (green) $N=12$, (red) $N=15$. Increasing the system size the discrepandy form the EX solution of the SCL decreases both for the homogeneous (top) and the inhomogeneous (bottom) case.

Beside the density profile it is worth mentionning that the SCL also captures the main features of the equal-time correlation functions. In Figs 22 and 23 we plot the momentum distribution obtained from the single particle density matrix $\left\langle\hat{b}_{i}^{\dagger}(t) \hat{b}_{j}(t)\right\rangle_{0}$ for two different times and interaction strenghts.

It can be seen that the SCL follows the behavior of the EX solution althoght showing a deviations at high momenta $k$. It
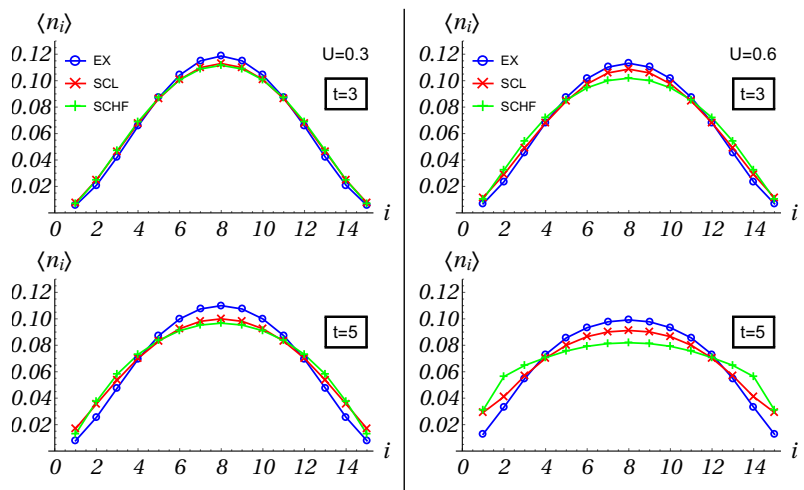

Figure 19: (Color online). Average number of bosons per site at two different times (upper row) $t=3$ and (lower row) $t=5$ and for two different final interactions (left column) $U=0.3$ and (right column) $U=0.6$. Here for a homogeneous system (see text) with $N=15$ sites, $n=5$ initially in its non-interacting ground state $U=0$.
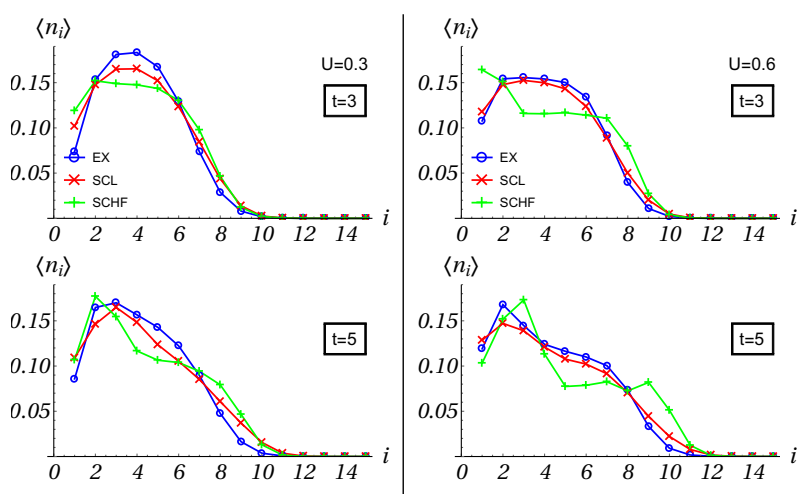

Figure 20: (Color online). Average number of bosons per site at two different times (upper row) $t=3$ and (lower row) $t=5$ and for two different final interactions (left column) $U=0.3$ and (right column) $U=0.6$. Here for an inhomogeneous system (see text) with $N=15$ sites, $n=5$ initially in its non-interacting ground state $U=0$.
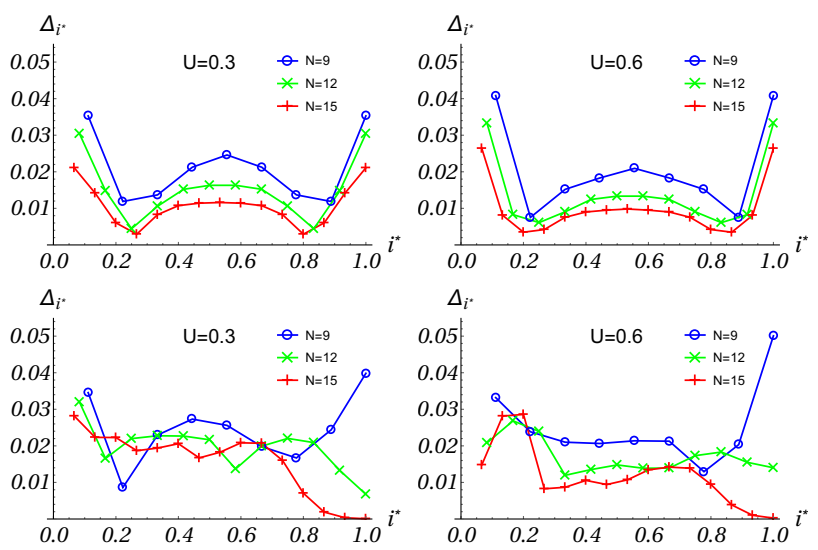

Figure 21: (Color online). Maximum deviation per site $\left(i^{*}=i / N\right)$ over total time interval beteewn the EX solution and the SCL for three different system sizes: (blue) $N=9$, (green) $N=12$ and (red) $N=15$. 

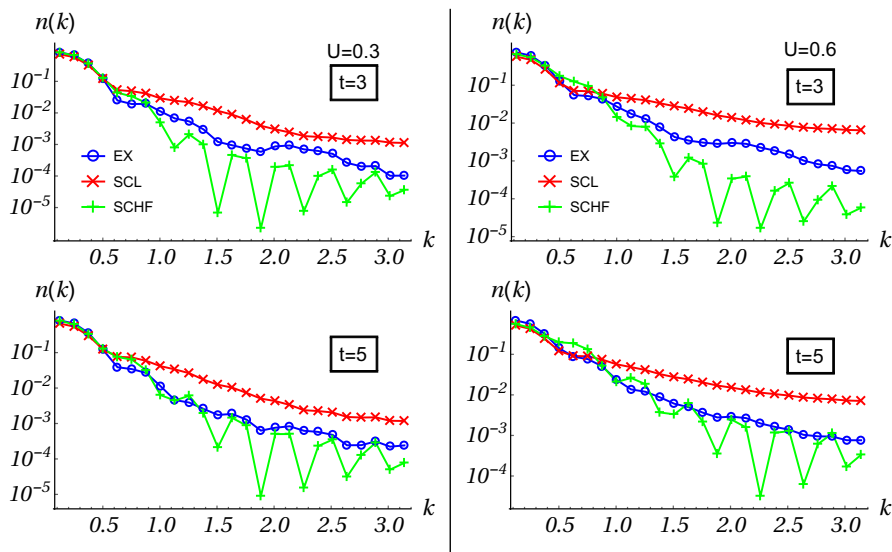

Figure 22: (Color online). Momentum distribution for the homogenous case and for a system with $N=15$ sites and $n=5$ bosons at two different times: (top) $t=3$ and (bottom) $t=5$ and for two different final interaction strenghts: (left) $U=0.3$ and (right) $U=0.6$.
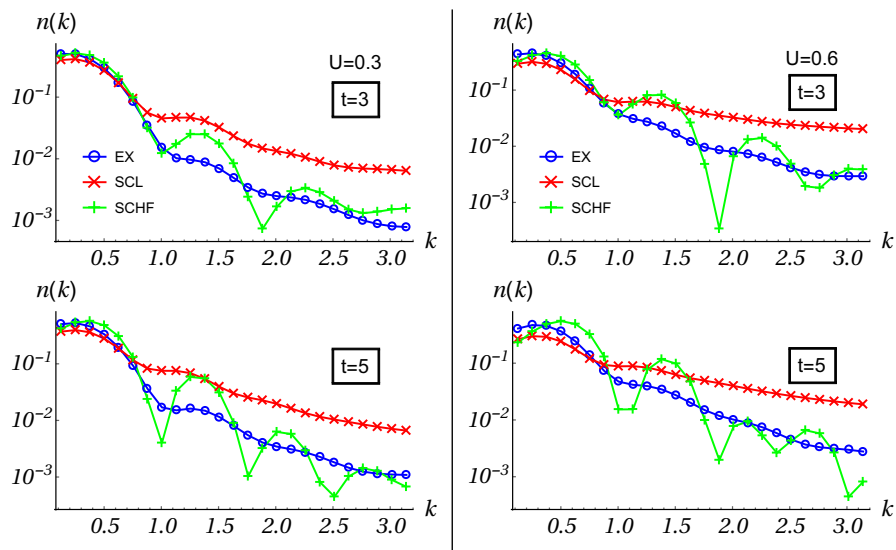

Figure 23: (Color online). Momentum distribution for the inhomogenous case (see text) and for a system with $N=15$ sites and $n=5$ bosons at two different times: (top) $t=3$ and (bottom) $t=5$ and for two different final interaction strenghts: (left) $U=0.3$ and (right) $U=0.6$. is interesting to observe that for the inhomogeneous case the SCL shows the "plasmonic"-like excitation around $k \approx 1.2$ which is manifested as a plateaux in $n(k)$. This is nothing but a density wave-packet traveling towards the right boundary.

In Fig 24 we show again the maximum deviation per momentum $k$ over the whole time evulution for different system sizes. It can be observed that the general behavior is that the maximum deviation decreases with sistem size.

Summarizing we can say that the comparison of the selfconsistent ladder with the exact diagonalization, at zero temperature and for small systems, shows that we can rely on this approach to describe the dynamics of bigger systems On the other hand, we can use the comparison at zero temperature to infer that there will be agreement also at finite temperature due to the fact that the total Hamiltonian does not couple subspaces of the Hilbert space with different total number of bosons. The dynamical quantities for an initial Gibbs state are the weighted average of quantities evolving in subspaces with
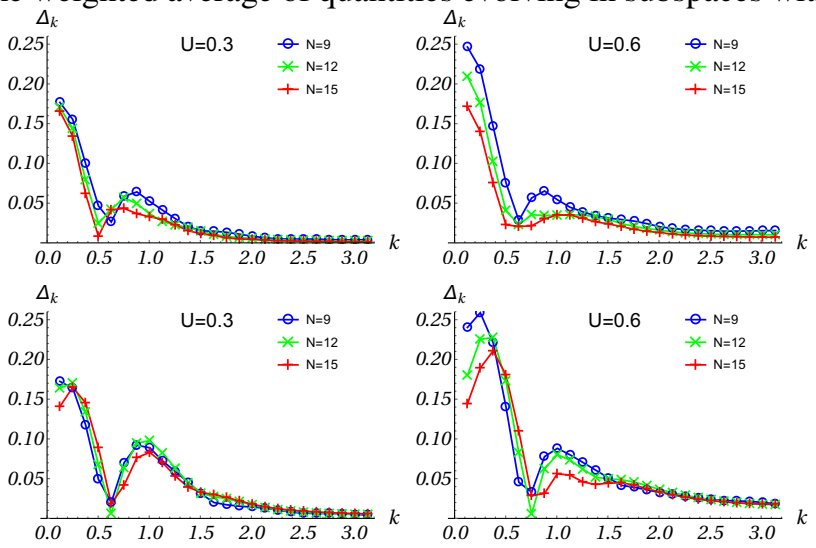

Figure 24: (Color online). Maximum deviation per momentum $k$ over total time interval beteewn the EX solution and the SCL for three different system sizes: (blue) $N=9$, (green) $N=12$ and (red) $N=15$.

fixed number of bosons.
1 A. Polkovnikov, K. Sengupta, A. Silva, and M. Vengalattore, Colloquium: Nonequilibrium dynamics of closed interacting quantum systems, Rev. Mod. Phys. . 83, 863 (2011).

2 J. Eisert, M. Friesdorf, and C. Gogolin, Quantum many-body systems out of equilibrium, Nature Physics 11, 124 (2015).

${ }^{3}$ R. Nandkishore, and D.A. Huse, Many-body localization and thermalization in quantum statistical mechanics, Annual Review of Condensed Matter Physics 6, 15 (2015).

4 J.-S. Wang, J. Wang, and N. Zeng, Nonequilibrium Greens function approach to mesoscopic thermal transport, Phys. Rev. B 74, 033408 (2006).

5 R. Vosk, and E. Altman, Dynamical quantum phase transitions in random spin chains, Phys. Rev. Lett. 112, 217204 (2014).

${ }^{6}$ R. Schützhold, M. Uhlmann, Y. Xu, and U.R. Fischer, Sweeping from the Superfluid to the Mott Phase in the Bose-Hubbard Model,
Phys. Rev. Lett. 97, 200601 (2006).

7 U.R. Fischer, R. Schützhold, and M. Uhlmann, Bogoliubov theory of quantum correlations in the time-dependent Bose-Hubbard model, Phys. Rev. A 77, 043615 (2008).

${ }^{8}$ C. Kollath, A.M. Läuchli, and E. Altman, Quench dynamics and nonequilibrium phase diagram of the Bose-Hubbard model, Phys. Rev. Lett. 98, 180601 (2007).

${ }^{9}$ I. Bloch, J. Dalibard, and W. Zwerger, Many-body physics with ultracold gases, Rev. Mod. Phys. 80, 885 (2008).

10 M.A. Cazalilla, R. Citro, T. Giamarchi, E. Orignac, and M. Rigol, One dimensional bosons: From condensed matter systems to ultracold gases, Rev. Mod. Phys. 83, 1405 (2011).

11 I. Bloch, J. Dalibard, and S. Nascimbéne, Quantum simulations with ultracold quantum gases, Nature Physics 8, 267 (2012).

12 M. P. A. Fisher, P. B. Weichman, G. Grinstein, and D. S. Fisher, 
Boson localization and the superfluid-insulator transition, Phys. Rev. B, 40, 546 (1989).

13 M.P. Kennett, Out-of-equilibrium dynamics of the Bose-Hubbard model, ISRN Condensed Matter Physics 2013, 393616 (2013).

14 M.P. Kennett, and D. Dalidovich, Schwinger-Keldysh approach to out-of-equilibrium dynamics of the Bose-Hubbard model with time-varying hopping, Phys. Rev. A 84, 033620 (2011).

15 M.R.C. Fitzpatrick, and M.P. Kennett, Space-time correlations in the Bose Hubbard model after a quantum quench, arXiv:1606.04117 (2016).

16 J.M. Zhang, and R.X. Dong, Exact diagonalization: the BoseHubbard model as an example, EJP 31, 591 (2010).

17 M.A. Cazalilla, and J.B. Marston, Time-dependent density-matrix renormalization group: a systematic method for the study of quantum many-body out-of-equilibrium systems, Phys. Rev. Lett. 88, 256403 (2002).

18 L.V. Keldysh, Diagrammatic technique for non-equilibrium processes, Sov. Phys. JETP 20, 1018 (1965).

19 A. Kamenev, Field theory of non-equilibrium systems, Cambridge University Press (2011).

${ }^{20}$ G. Stefanucci and R. van Leeuwen, Nonequilibrium Many-Body Theory of Quantum Systems, Cambridge University Press (2013).
${ }^{21}$ R. Starke and G. Kresse, Self-consistent Green function equations and the hierarchy of approximations for the four-point propagator, Phys. Rev. B 85, 075119 (2012).

22 A. Schindlmayr and R.W. Godby, Systematic Vertex Corrections through Iterative Solution of Hedin's Equations Beyond the GW Approximation, Phys. Rev. Lett. 80, 1702 (1998).

23 R.D. Mattuck, A guide to Feynman diagrams in many-body problem, McGraw-Hill (1967).

24 A.L. Fetter and J.D. Walecka, Quantum theory of many-particle systems, McGraw-Hill (1980).

25 G. Baym and L. P. Kadanoff, Conservation laws and correlation functions, Phys. Rev. 124, 287 (1961).

26 N. Lo Gullo and L. Dell'Anna, Spreading of correlations and Loschmidt echo after quantum quenches of a Bose gas in the Aubry-André potential, Phys. Rev. A 92, 063619 (2015).

27 J.P. Ronzheimer et al, Expansion dynamics of interacting bosons in homogeneous lattices in one and two dimensions, Phys. Rev. Lett. 110, 205301 (2013).

${ }^{28}$ G. Carleo, et al, Light-cone effect and supersonic correlations in one- and two dimensional bosonic superfluids, Phys. Rev. A 89, 031602(R) (2014). 